\title{
CONTRIBUTIONS OF BOTANISTS OF NATURAL HISTORY MUSEUM (TRIBHUVAN UNIVERSITY) TO RESEARCH AND EDUCATION IN NEPAL
}

\author{
DHARMA RAJ DANGOL \\ Natural History Museum, Institute of Science and Technology, Tribhuvan University \\ Swayambhu, Kathmandu, Nepal \\ drdangol@gmail.com
}

\begin{abstract}
This paper deals with the contribution of botanists of Natural History Museum,Tribhuvan University, Nepal in the field of plant research and education. Of the 14 listed, only two botanists are continuing their service in the museum. Their research works mainly focused on collection and exploration of flora, supervision of dissertation including publication of 171 articles in different journals of national and international repute. Apart from this, their contributions in education/training by organizing seminars/trainings/workshops are highly significant.
\end{abstract}

Keywords: botany education/training, plant research, plant specimen collection, thesis research

\section{INTRODUCTION}

Natural History Museum (NHM) was established by Tribhuvan University, the pioneer University of Nepal, under it's Institute of Science (Currently, Institute of Science and Technology) on July 17, 1975 at the foothill of Swayambhu, the World Heritage Site. This museum is located approximately $5 \mathrm{~km}$ west from Kathmandu Metropolitan City. Basically, this museum was proposed to: (i) house Nepalese flora, fauna and paleontological specimens; (ii) conduct research and education activities in Nepal for generating data and conservation of precious natural resources; and (iii) prepare documents related to natural history of Nepal.

This museum is the scientific authority for the fauna of CITES (Convention on International Trade on Endangered Species of Wild Fauna and Flora) in Nepal (TU Today, 2015). This museum now has its collections of more than 55,700 specimens of plants, animals, and fossils (TU Today, 2015). Some of the interesting specimens that visitors love to see include two headed snake, king cobra, 20'4" long exuviae of python, molar teeth and skull of Archidiscodon (3 to 1 million years old), molar teeth of Ramapithecus (11 million years old), skull and lower jaw of Hippopotamus, embryos (of human, elephant and rhinoceros), different species of Cordyceps, insectivorous plants, samples of medicines and artefact collections (Dangol, 2013).

One of the significant publications of this museum is the Journal of Natural History Museum which is brought out annually since 1977 and includes original research findings on different aspects of flora, fauna and geology of the country. Besides this, it has also published several books and field guide books based on the research and experiences. This museum is also offering opportunities to all interested student volunteers of national and international levels, 
faculties and researchers for the shorter or longer periods to work with the scientists of the museum in the field of flora, fauna and palaeontology of Nepal. Botanists and zoologists are working significantly to achieve the goals and objectives of this museum through thier research, publications and education/trainings. The purpose of this paper is to highlight the contribution made by the botanists of $\mathrm{NHM}$ focussing their significant research and educational activities.

\section{MATERIALS AND METHODS}

A preliminary study was done to collect the list of botanists who are currently working or had worked in the Natural History Museum and was done by consulting the yearly register books of the museum and General Administrative Office of Tribhuvan University, Kirtipur. To obtain data of their collections, the register books of plant collections were consulted. Moreover, to record the research projects and publications (books, journal articles, popular articles, proceedings papers, abstracts, research reports), libraries of NHM and TU, internets (Google scholar) and websites (www.nhmnepal.edu.np) were visited and were verified accurately. An annex of the publications of the botanists of the museum was prepared for developing database with published literatures. Official documents were reviewed and administration staffs of the Natural History Museum were consulted to record the contribution made in the sector of education and training from its establishment period to date.

\section{RESULTS AND DISCUSSION}

\section{State of botanists in the Museum}

Natural History Museum has employed botanists and zoologists as its faculty to conduct research in natural science discipline. If we look the table 1, there is the list of 14 faculties now working or have worked in the museum as botanists. Two botanists (Keshab Shrestha and Hemant Ram Bhandari) worked for more than 2 decades. Most of other botanists have worked for a shorter period and left the museum to join Amrit Science Campus (Devendra Mananda Bajracharya and Roshani Manandhar) or Trichandra Campus (Anjali Maiya Shrestha and Suman Bhattarai) or Central Department of Botany (Mohan Siwakoti) or RECAST (Shilesh Chandra Singh and Shova Devi Shrestha). Nirmala Pradhan and Dharma Raj Dangol are only the two botanists currently working in museum (table 1). 
TABLE 1. Botanists of Natural History Museum and their position and service year.

\begin{tabular}{|c|c|c|c|c|c|}
\hline SN & Name & Position ${ }^{7}$ & $\begin{array}{l}\text { Service year } \\
\text { in NHM }\end{array}$ & Start & End \\
\hline \multirow{2}{*}{1} & \multirow{2}{*}{ Shailesh Chandra Singh, Ph.D. ${ }^{1}$} & Reader & 4 & 2032.4 .1 & 2036.12 .5 \\
\hline & & Professor & 4 & 2051.6 .6 & 2055.6 .5 \\
\hline 2 & Nirmal Kumar Bhattarai ${ }^{6}$ & $\begin{array}{l}\text { Assistant } \\
\text { lecturer }\end{array}$ & 3 months & 2034.1 .2 & 2034.3.30 \\
\hline 3 & Hemant Ram Bhandari ${ }^{2}$ & Lecturer & 21 & 2037.3 .23 & 2058.1 .30 \\
\hline 4 & Roshani Manandhar ${ }^{4}$ & $\begin{array}{l}\text { Assistant } \\
\text { lecturer }\end{array}$ & 2 & 2038.7 .24 & 2040.8 .1 \\
\hline 5 & Suman Bhattarai ${ }^{3}$ & $\begin{array}{l}\text { Assistant } \\
\text { lecturer }\end{array}$ & 2 & 2038.9 .24 & 2040.7 .23 \\
\hline 6 & Anjali Maiya Shrestha ${ }^{3}$ & $\begin{array}{l}\text { Assistant } \\
\text { lecturer }\end{array}$ & 3 & 2040.8 .1 & 2043.8.20 \\
\hline 7 & Devendra Mananda Bajracharya ${ }^{4}$ & $\begin{array}{l}\text { Assistant } \\
\text { lecturer }\end{array}$ & 2 years & 2041 & 2042 \\
\hline 8 & $\begin{array}{l}\text { Baidya Nath Upadhyaya } \\
\text { (Asst. Research Officer) }\end{array}$ & $\begin{array}{l}\text { Assistant } \\
\text { lecturer }\end{array}$ & 3 years & 2033.12 .21 & 2036.10 .1 \\
\hline 9 & Shova Devi Shrestha & $\begin{array}{l}\text { Assistant } \\
\text { lecturer }\end{array}$ & 3 years & 2033.10 .15 & 2036.8.24 \\
\hline 10 & Mohan Siwakoti ${ }^{5}$ & Reader & 4 years & 2060.9 .18 & 2064.9.11 \\
\hline 11 & Mahesh Adhikari ${ }^{1}$ & $\begin{array}{l}\text { Associate } \\
\text { Professor }\end{array}$ & 1 year & 2066.11 .9 & 2067.3.30 \\
\hline 12 & $\begin{array}{l}\text { Keshab Shrestha, Ph.D. }{ }^{1} \\
\text { (Asst. Research Officer) }\end{array}$ & Professor & 34 & 2035.5 .7 & 2069 \\
\hline 13 & Nirmala Pradhan, Ph.D. & Professor & 32 & 2040.7 .23 & To date \\
\hline 14 & Dharma Raj Dangol & Professor & 3 & 2069.6 .5 & To date \\
\hline
\end{tabular}

1= Retired; $2=$ Left the country for Canada; $3=$ Tri-Chandra Multiple Campus; $4=$ Amrit Science Campus; $5=$ Central Department of Botany; $6=$ Passed away; $7=$ POSITION OF BOTANISTS DURING THEIR SERVICE PERIOD AT NHM

\section{Contributions to plant research}

\section{Research projects}

Zoologists and botanists of this museum also developed and conducted research projects in different parts of Nepal (table 2). These research projects were supported by Pro-Natura Fund Japan, IOST, RONAST, WWF, and IUCN. Some of the projects were jointly carried out with international scientists (Appendix 1). 
TABLE 2. Completed and on-going key research projects.

\begin{tabular}{|c|c|c|c|}
\hline Year & Researchers & Report/Project titles & Reports submitted to \\
\hline 1982 & $\begin{array}{l}\text { Hagiwara, H and } \\
\text { Bhandary, H R }\end{array}$ & $\begin{array}{l}\text { Myxomycetes from central } \\
\text { Nepal. Reports on the } \\
\text { cryptogamic study in Nepal. }\end{array}$ & $\begin{array}{l}\text { Miscellaneous } \\
\text { publication of the } \\
\text { National Science } \\
\text { Museum, Tokyo }\end{array}$ \\
\hline 1985 & $\begin{array}{l}\text { Wilson, E; } \\
\text { Walisiewicz, M; } \\
\text { Harvey, S; Gay, H } \\
\text { and Shrestha, K }\end{array}$ & $\begin{array}{l}\text { The Report of the Oxford } \\
\text { University expedition to Nepal. }\end{array}$ & Oxford University \\
\hline 1996 & $\begin{array}{l}\text { Singh, S C; Shrestha, } \\
\mathrm{K} \text { and Bhandary, H R }\end{array}$ & $\begin{array}{l}\text { A study of some rare, } \\
\text { endangered and endemic } \\
\text { plants of central Nepal (Draft } \\
\text { report) }\end{array}$ & NHM-TU \\
\hline 1997 & $\begin{array}{l}\text { Singh, S C and H.R. } \\
\text { Bhandary }\end{array}$ & $\begin{array}{l}\text { Establishment of computerized } \\
\text { data base in the Natural History } \\
\text { Museum (Draft report). }\end{array}$ & NHM-TU \\
\hline 1998 & $\begin{array}{l}\text { Karki, J B; Shrestha, } \\
\text { K and Khanal, B }\end{array}$ & $\begin{array}{l}\text { Study on potential of } \\
\text { biodiversity conservation of } \\
\text { Badimalika region of Achham, } \\
\text { Bajura, Bajhang and Kalikot } \\
\text { districts of Nepal. } \\
\end{array}$ & $\begin{array}{l}\text { Nepal Biodiversity Action } \\
\text { Plan, Kathmandu }\end{array}$ \\
\hline 1998 & $\begin{array}{l}\text { Shrestha, K; Khanal, } \\
\text { B and Karki; J B }\end{array}$ & $\begin{array}{l}\text { Inventory of biodiversity in } \\
\text { Badimalika and Rama-Roshan } \\
\text { region, west Nepal. }\end{array}$ & $\begin{array}{l}\text { National Biodiversity } \\
\text { Action Plan, Kathmandu }\end{array}$ \\
\hline 2000 & $\begin{array}{l}\text { Pradhan, N; } \\
\text { Shrestha, K; Shah, K } \\
\text { B; Shrestha, P K and } \\
\text { Khanal, B }\end{array}$ & $\begin{array}{l}\text { Study on the biodiversity of } \\
\text { Swayambhu area }\end{array}$ & IOST-TU \\
\hline 2000 & $\begin{array}{l}\text { Shrestha, P K and } \\
\text { Pradhan, N }\end{array}$ & $\begin{array}{l}\text { A general survey of invertebrate } \\
\text { (except Lepidoptera) in Royal } \\
\text { Bardia National Park, Nepal. }\end{array}$ & $\begin{array}{l}\text { World Wildlife Fund } \\
\text { (WWF) Nepal Program, } \\
\text { Kathmandu, Nepal }\end{array}$ \\
\hline 2001 & $\begin{array}{l}\text { Khanal, B; Shrestha, } \\
\text { P K and Shrestha, K }\end{array}$ & $\begin{array}{l}\text { Study of insect fauna of tropical } \\
\text { region of Nepal and their } \\
\text { conservation measures (Final } \\
\text { Report). }\end{array}$ & $\begin{array}{l}\text { Pro-Natura Fund, } \\
\text { Japan/NCSJ, } \\
\text { 134+Maps+photographs }\end{array}$ \\
\hline 2001 & $\begin{array}{l}\text { Shrestha, K; Khanal, } \\
\text { B and Shrestha, P K }\end{array}$ & $\begin{array}{l}\text { Insect fauna and their } \\
\text { conservation in tropical Nepal. }\end{array}$ & $\begin{array}{l}\text { Pro-Natura Fund, } \\
\text { Japan10:145-159. }\end{array}$ \\
\hline
\end{tabular}




\begin{tabular}{|c|c|c|c|}
\hline 2001 & $\begin{array}{l}\text { Shrestha, K; Khanal, } \\
\text { B and Karki, J B }\end{array}$ & $\begin{array}{l}\text { Floral diversity in Badimalika } \\
\text { and Rama-Roshan region, mid- } \\
\text { west Nepal. }\end{array}$ & DNPWC \\
\hline 2003 & $\begin{array}{l}\text { Shrestha, K; Adhikari, } \\
\text { B; Allen, M; Courage, } \\
\text { A; Hall, H; Khanal, } \\
\text { B; Koirala, S; Kunwar, } \\
\text { R; Malicky, H; } \\
\text { Pariya, B; Pradhan, } \\
\text { N; Shah, K; Shakya, } \\
\text { S; Sharma, S; Shaw, } \\
\text { A; Shrestha, K; } \\
\text { Shrestha, P K; Smith, } \\
\text { C and Zimmerman, B }\end{array}$ & $\begin{array}{l}\text { Babai river valley fish and } \\
\text { biodiversity survey: Higher } \\
\text { plants. In: S. Oliver (ed.), } \\
\text { Babai River Valley Fish and } \\
\text { Biodiversity Survey: Royal } \\
\text { Bardia National Park, Nepal. }\end{array}$ & $\begin{array}{l}\text { ZSL Conservation } \\
\text { Report, Zoological } \\
\text { Society of London, pp. } \\
\text { 39-48. }\end{array}$ \\
\hline 2004 & Pradhan, N & $\begin{array}{l}\text { Bryoflora of Chitwan, central } \\
\text { Nepal Final report on study } \\
\text { of the biodiversity of tropical } \\
\text { region of Nepal (Chitwan } \\
\text { district). pp. 48-61. }\end{array}$ & Pro-Natura Fund, Japan \\
\hline 2004 & $\begin{array}{l}\text { Shrestha, K; Shrestha, } \\
\text { P K; Khanal, B and } \\
\text { Shakya. S }\end{array}$ & $\begin{array}{l}\text { Study of the biodiversity of the } \\
\text { tropical region of Nepal (mid- } \\
\text { term report). }\end{array}$ & Pro-Natura Fund, Japan \\
\hline 2004 & $\begin{array}{l}\text { Shrestha, K; } \\
\text { Shrestha, P K; Khanal, } \\
\text { B; Pradhan, N and } \\
\text { Shakya, S }\end{array}$ & $\begin{array}{l}\text { Study of the biodiversity } \\
\text { of tropical region of Nepal } \\
\text { (Chitwan district) } \\
\text { (Final report). }\end{array}$ & $\begin{array}{l}\text { Pro-Natura Fund, Japan } \\
\text { submitted by CENEED/ } \\
\text { NHM-TU }\end{array}$ \\
\hline 2005 & $\begin{array}{l}\text { Shrestha, K; } \\
\text { Shrestha, P K; } \\
\text { Khanal, B; Pradhan, N } \\
\text { and Shakya, S }\end{array}$ & $\begin{array}{l}\text { Study on the species diversity } \\
\text { in the Chitwan district of central } \\
\text { Nepal. }\end{array}$ & $\begin{array}{l}\text { Pro-Natura Fund, Annua } \\
\text { report, 14:175-192 }\end{array}$ \\
\hline 2008 & $\begin{array}{l}\text { Shrestha-Vaidya, } \\
\text { G;Shrestha, K and } \\
\text { Wallander, H }\end{array}$ & $\begin{array}{l}\text { Evaluation of the antimicrobial } \\
\text { activity of Lantana camara Linn. } \\
\text { against pathogenic fungi and } \\
\text { bacteria. }\end{array}$ & $\begin{array}{l}\text { NAST, Khumaltar, and } \\
\text { Microbial Ecology Lab, } \\
\text { Lund University, Sweden }\end{array}$ \\
\hline 2043 & Shrestha, K & $\begin{array}{l}\text { Study of the natural } \\
\text { environment of Dang }\end{array}$ & RONAST \\
\hline 2015 & Dangol, D R & $\begin{array}{l}\text { Establishment of plant genetic } \\
\text { resources in NHM } 2014 \text { July- } \\
2015 \text { Feb. }\end{array}$ & $\begin{array}{l}\text { LIBIRD \& Bioversity } \\
\text { International }\end{array}$ \\
\hline
\end{tabular}




\begin{tabular}{|l|l|l|l|}
\hline $\begin{array}{l}\text { 2015- } \\
2016\end{array}$ & $\begin{array}{l}\text { Joshi, N; Shrestha, R; } \\
\text { Basnet, R; Dangol, D } \\
\text { R and Pradhan, N }\end{array}$ & $\begin{array}{l}\text { Revision of flora of Phulchowki } \\
\text { and Godawari. }\end{array}$ & DPR \\
\hline 2015- & $\begin{array}{l}\text { Dangol, D R; } \\
\text { Pradhan, N; Shrestha, } \\
\text { R; Joshi, N and } \\
\text { Basnet, R (2015- } \\
\text { 2017) }\end{array}$ & Swayambhu Flora Project. & IOST and DPR \\
\hline
\end{tabular}

\section{Ph.D. research}

Two botanists Keshab Shrestha and Nirmala Pradhan completed their Doctor of Philosophy after joining NHM. Keshab Shrestha did his Ph.D. research on physioecological study of algae from Kyoto University of Japan in 1993 and Nirmala Pradhan did her Ph.D. on bryoflora of terai and churia hills of lowland Nepal from Tribhuvan University of Nepal in 2010 (table 3).

TABLE 3. Botanists of Natural History Museum with their Ph.D. thesis research and degree awarding universities and completion year.

\begin{tabular}{|l|l|l|l|l|}
\hline SN & Researcher & Thesis Research & University & $\begin{array}{l}\text { Completion } \\
\text { year }\end{array}$ \\
\hline 1 & Keshab Shrestha & $\begin{array}{l}\text { Physioecological study on fresh } \\
\text { water chlorophyte Gonium } \\
\text { pectorale isolated from Tibet } \\
\text { (China), Nepal and the Ryukyu } \\
\text { Islands (Japan) }\end{array}$ & $\begin{array}{l}\text { Kyoto University, } \\
\text { Japan }\end{array}$ & 1993 \\
\hline 2 & $\begin{array}{l}\text { Dharma Raj } \\
\text { Dangol }\end{array}$ & $\begin{array}{l}\text { Agrestal weeds of Nepal: } \\
\text { Identification, distribution, ecology } \\
\text { and agricultural importance }\end{array}$ & $\begin{array}{l}\text { Vienna } \\
\text { University, } \\
\text { Austria }\end{array}$ & 1998 \\
\hline 3 & Nirmala Pradhan & $\begin{array}{l}\text { Bryophytes of Lowland Nepal: } \\
\text { Tarai and Churia Hills }\end{array}$ & $\begin{array}{l}\text { Tribhuvan } \\
\text { University, Nepal }\end{array}$ & 2010 \\
\hline
\end{tabular}

${ }^{1}$ Ph.D. before joining Natural History Museum.

\section{Thesis supervised by botanists of NHM}

Faculty members of NHM supervised M.Sc. and Ph.D. dissertations (table 4). Prof Keshab Shrestha had supervised two Ph.D. students, one from Tribhuvan University and another from Nepal Academy of Science and Technology. Dharma Raj Dangol also supervised one MSc. student from School of Environmental Science and Management (SchEM) affiliated with Pokhara University, 
TABLE 4. Botanists of Natural History Museum as supervisors of M.Sc. and Ph.D. dissertations.

\begin{tabular}{|l|l|l|l|}
\hline SN & $\begin{array}{l}\text { Faculty members } \\
\text { of NHM }\end{array}$ & $\begin{array}{l}\text { Name of the Ph.D. and M.Sc. students } \\
\text { names and their titles of dissertations }\end{array}$ & $\begin{array}{l}\text { Year of } \\
\text { Completion }\end{array}$ \\
\hline 1 & Keshab Shrestha & $\begin{array}{l}\text { Ila Shrestha', Ph.D. in Botany } \\
\text { Ethnobotany of Tamang Community in } \\
\text { Langtang National Park and Buffer Zone, } \\
\text { Central Nepal, with Application of Geographic } \\
\text { Information System and Remote System }\end{array}$ & 2010 \\
\cline { 2 - 4 } & $\begin{array}{l}\text { Geeta Shrestha-Vaidya', Ph.D. in Botany } \\
\text { Influence of mycorrhizal fungi in stabilizing soils } \\
\text { subjected to Erosion in Nepal }\end{array}$ & 2010 \\
\hline 2 & $\begin{array}{l}\text { Saraswati Bhurtyal }{ }^{3}, \text { M.Sc. in Environmental } \\
\text { Danagement } \\
\text { Dharma Raj Dangol } \\
\text { Market System of Smallholder Farmers around } \\
\text { Pokhara: A Case Study of Bhadaure Tamagi } \\
\text { VDC of Kaski District of Nepal }\end{array}$ & 2013 \\
\hline
\end{tabular}

${ }^{1}$ Patan Multiple Campus, Tribhuvan University, Patan, Lalitpur

${ }^{2}$ Nepal Academy of Science and Technology, Khumaltar, Lalitpur

${ }^{3}$ School of Environmental Science and Management, Pokhara University, Nayabaneshwar, Kathmandu

\section{Collections of plant specimens}

Natural History Museum houses botanical specimens of algae, fungi and mushrooms, lichens, bryophytes, pteridophytes, gymnosperms, and angiosperms made by the plant collectors, botanists of $\mathrm{NHM}$ and obtained from other institutions (table 5). It has also deposited historical herbaria made by three plant collectors of Nepal (Shrestha \& Dahal, 2010). All the specimens are preserved well following dry and wet preservation methods. It has some collection of insectivorous plants, healing plants, wild food plants and four species of Cordyceps. It also has artefact in its collections contributed by different ethnic groups of Nepal and Japan. 
TABLE 5. Plant collections, preservation types and collectors.

\begin{tabular}{|c|c|c|c|c|}
\hline \multirow[t]{2}{*}{ SN } & \multirow{2}{*}{$\begin{array}{l}\text { Groups of } \\
\text { botanical } \\
\text { specimens }\end{array}$} & \multirow[t]{2}{*}{ Preservation types } & \multicolumn{2}{|c|}{ Plant collectors } \\
\hline & & & NHM botanists & $\begin{array}{l}\text { Non-NHM } \\
\text { botanists }\end{array}$ \\
\hline 1 & Algae & Dry preservation & --- & Gift from Japan \\
\hline 2 & Fungi & Wet preservation & $\begin{array}{l}\text { Shailesh Chandra } \\
\text { Singh, Hemanta Ram } \\
\text { Bhandhary }\end{array}$ & Hari Prasad Aryal \\
\hline 3 & Lichens & Dry preservation & --- & |-- \\
\hline 4 & Bryophytes & $\begin{array}{l}\text { Dry \& wet } \\
\text { preservations }\end{array}$ & Nirmala Pradhan & $\mid--$ \\
\hline 5 & Pteridophytes & $\begin{array}{l}\text { Herbarium and wet } \\
\text { preservation }\end{array}$ & $\begin{array}{l}\text { Keshab Shrestha, } \\
\text { Nirmala Pradhan }\end{array}$ & --- \\
\hline 6 & Gymnosperms & $\begin{array}{l}\text { Herbarium, dry and } \\
\text { wet preservation }\end{array}$ & Mohan Siwakoti & --- \\
\hline 7 & Angiosperms & $\begin{array}{l}\text { Herbarium, dry and } \\
\text { wet preservation }\end{array}$ & $\begin{array}{l}\text { Keshab Shrestha, } \\
\text { Nirmala Pradhan, } \\
\text { Hemant Ram Bhandary, } \\
\text { Anjali Maiya Shrestha, } \\
\text { Dharma Raj Dangol }\end{array}$ & $\begin{array}{l}\text { Purusottam } \\
\text { Shrestha, Ila } \\
\text { Shrestha }\end{array}$ \\
\hline
\end{tabular}

\section{Contributions to scientific literature}

A list of 171 publications of botanists working in Natural History Museum were grouped into abstracts (5), books and booklets (12), edited book chapters (10), journal papers (105), popular articles (74), proceeding papers (19) and research reports (19) (Appendix 1).

SC Singh wrote papers in co-authorship with all NHM botanists, whereas some botanists published individually or in co-authorship with zoologists or botanists of other institutions (table 6 and 7).

A total of 29 papers were single authored whereas, 86 papers double or multi-authored papers (table 7). In case of double or multi-authored papers, lead authored papers (35) by the botanists were fewer in number to co-authored papers (51). Of the total papers (53), published by Keshab Shrestha, 11 papers were single authored, 10 papers lead authored and 32 papers co-authored (table7). 
TABLE 6. Authorship analysis of double or multi-authored journal papers.

\begin{tabular}{|c|c|c|c|c|c|c|c|c|c|c|c|}
\hline Lead authors (double or multiple) & $\begin{array}{l}\mathscr{U} \\
\text { ON }\end{array}$ & $\underline{w}$ & 只 & $\begin{array}{l}\stackrel{m}{\underline{\alpha}} \\
\frac{1}{I}\end{array}$ & 只 & 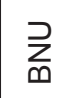 & $\sum_{<}^{\infty}$ & $\sum^{\infty}$ & 只 & $\underset{\mathbf{Z}}{\bar{Z}}$ & $\sum_{z}^{N}$ \\
\hline Shailesh Chandra Singh (SCS) & $x$ & $\sqrt{ }$ & $\sqrt{ }$ & $\sqrt{ }$ & & $\sqrt{ }$ & & & & $\sqrt{ }$ & $\sqrt{ }$ \\
\hline Keshab Shrestha (KS) & & $\mathrm{X}$ & & & & & & & & $\sqrt{ }$ & $\sqrt{ }$ \\
\hline Nirmala Pradhan (NP) & & & $\mathrm{x}$ & & & & & & & & $\sqrt{ }$ \\
\hline Hemanta Ram Bhandary (HRB) & & & & $x$ & & & & & & & $\sqrt{ }$ \\
\hline Shova Devi Shrestha (SDS) & & & & & $x$ & & & & & & \\
\hline Baidya Nath Upadhyaya (BNU) & & & & & & $x$ & & & & & \\
\hline Anjali Maiya Shrestha (AMS) & & & & & & & $x$ & & & & \\
\hline Mohan Siwakoti (MS) & & & & & & & & $x$ & & & $\sqrt{ }$ \\
\hline Dharma Raj Dangol (DRD) & & & & & & & & & $x$ & & $\sqrt{ }$ \\
\hline NHM Zoologists (NZ) & & & & & & & & & & $x$ & \\
\hline Non-NHM writers (NNW) & & & & & & & & & & & $x$ \\
\hline
\end{tabular}

TABLE 7. Authorship analysis of refereed journal papers.

\begin{tabular}{|c|c|c|c|c|c|}
\hline \multirow{2}{*}{ SN } & \multirow{2}{*}{ NHM Botanists } & \multirow{2}{*}{$\begin{array}{l}\text { Single } \\
\text { authored }\end{array}$} & \multicolumn{2}{|c|}{ Double or multi-authored papers } & \multirow{2}{*}{ Total } \\
\hline & & & Lead author & Co-author & \\
\hline 2 & Keshab Shrestha & 11 & 10 & 32 & 53 \\
\hline 3 & Nirmala Pradhan & 7 & 9 & 3 & 18 \\
\hline 4 & Hemant Ram Bhandary & 2 & 4 & 7 & 13 \\
\hline 9 & Dharma Raj Dangol & 1 & 3 & 7 & 11 \\
\hline 1 & Shailesh Chandra Singh & 1 & 7 & 1 & 9 \\
\hline 8 & Mohan Siwakoti & 4 & 2 & & 6 \\
\hline 6 & Baidya Nath Upadhyaya & 2 & & 1 & 3 \\
\hline 5 & Shova Devi Shrestha & 1 & & & 1 \\
\hline \multirow[t]{2}{*}{7} & Anjali Maiya Shrestha & 1 & & & 1 \\
\hline & Total & 29 & 35 & 51 & 115 \\
\hline
\end{tabular}

Botanists of NHM published their scientific findings jointly with colleagues or other researchers of the university and research organizations (Appendix 1).

A total of 105 research articles in 29 scientific journals of six countries were published by 
botanists of NHM (table 8). Of the 29 journals, 17 were from Nepal, 5 were from Japan, each 2 were from France, India and USA, and one article published from China has been tabulated in table 8. Altogether the highest numbers of papers were published from Nepal in the Journal of Natural History Museum (54 papers), Scientific World (8 papers), Journal of Institute of Science and Technology (5 papers), and Our Nature (4 papers). Of the total 90, 15 papers were published in international journals and only six papers were first authored by botanists of NHM (Keshab Shrestha: 2, Nirmala Pradhan: 2, Sailesh Chandra Singh: 1 and Mohan Siwakoti: 1). Rest of the international papers were co-authored with Japanese and Nepalese researchers (Appendix 1).

TABLE 8. Alphabetical list of scientific journals/bulletins, name of the countries and number of papers published.

\begin{tabular}{|c|l|l|c|}
\hline SN & Name of the journals or bulletins & Countries & $\begin{array}{l}\text { Total } \\
\text { number } \\
\text { of papers }\end{array}$ \\
\hline 1 & Bamboo Journal & Japan & 1 \\
\hline 2 & Banko Jankari & Nepal & 1 \\
\hline 3 & Botanica Orientalis & Nepal & 2 \\
\hline 4 & Bulletin of Japanese Society of Microbial Ecology & Japan & 4 \\
\hline 5 & Bulletin of Pure and Applied Sciences & India & 1 \\
\hline 6 & Cryptogamie Bryologie & France & 1 \\
\hline 7 & Geobios & India & 1 \\
\hline 8 & Himalayan Biodiversity & Nepal & 2 \\
\hline 9 & International Journal of Applied Sciences and Biotechnology & Nepal & 1 \\
\hline 10 & International Journal of Environment & Nepal & 1 \\
\hline 11 & Japanese Journal of Limnology & Japan & 1 \\
\hline 12 & Journal of Ethnobiology and Ethnomedicine & USA & 1 \\
\hline 13 & Journal of Institute of Science and Technology & Nepal & 5 \\
\hline 14 & Journal of Japanese Botany & Japan & 1 \\
\hline 15 & Journal of Natural History Museum & Nepal & 54 \\
\hline 16 & Journal of Phycology & Japan & 1 \\
\hline 17 & Journal of Science (Trichandra College Science Association) & Nepal & 1 \\
\hline 18 & Museum & France & 1 \\
\hline
\end{tabular}




\begin{tabular}{|c|l|l|c|}
\hline 19 & Nepal Journal of Plant Sciences & Nepal & 2 \\
\hline 20 & Nepal Journal of Science and Technology & Nepal & 3 \\
\hline 21 & Nepalese Journal of Agricultural Sciences & Nepal & 1 \\
\hline 22 & Nepalese Journal of Biosciences & Nepal & 2 \\
\hline 23 & Our Nature & Nepal & 4 \\
\hline 24 & Plant Resources & Nepal & 1 \\
\hline 25 & Restoration Ecology & USA & 1 \\
\hline 26 & Scientific World & Nepal & 8 \\
\hline 27 & The Journal of Agriculture and Environment & Nepal & 1 \\
\hline 28 & The Wildlife & Nepal & 1 \\
\hline 29 & Wetland Science & China & 1 \\
\hline & Total & 6 & 105 \\
\hline
\end{tabular}

\section{Contributions to botany education/training after November 2012}

The botanists of Natural History Museum have contributed to curriculum development of new programs of the Institute of Science and Technology (B. Sc. in Tea Technology and Management) and Faculty of Education (M. Ed. in Biology). They delivered lectures on bryophytes in the Central Department of Botany, Tribhuvan University or "plant taxonomy" and "ethnobiology" in the Sanothimi Campus of Faculty of Education. Sometimes, botanists of the museum also helped to the postgraduate students of Central Department of Botany, Tribhuvan University in identification of bryophytes collected by them (PK Jha, 2013, Personal Communication). They delivered their lectures on special topics like in the CITES awareness programs, International Biodiversity Day (May 22), Environmental Day (June 5) and other events (table 9). They also delivered their lectures on the plant taxonomy, plant and bryophyte collection and ethnobiology. Introduction of NHM and agro-ecotourism in the seminars upon the demand of the students of Bachelor and Master Degrees was also managed. 
TABLE 9. Key educational events, topics covered, participant's organizations and level with numbers (2012 December-2015 December).

\begin{tabular}{|c|c|c|c|}
\hline $\begin{array}{l}\text { Event, Date } \\
\text { and venue }\end{array}$ & Topics covered & $\begin{array}{l}\text { Participants' } \\
\text { organizations }\end{array}$ & Level (Nr.)' \\
\hline $\begin{array}{l}\text { Seminar } \\
23.12 .2012 \\
\text { NHM }\end{array}$ & Plant research & $\begin{array}{l}\text { Patan Multiple Campus, } \\
\text { Tribhuvan University }\end{array}$ & $\begin{array}{l}\text { BSc Env Sci \& Botany } \\
(\mathrm{S}: 21, \mathrm{~T}: 2)\end{array}$ \\
\hline \multirow{2}{*}{$\begin{array}{l}\text { Seminar } \\
18.01 .2013 \\
\text { NHM }\end{array}$} & Bryophytes & \multirow{2}{*}{$\begin{array}{l}\text { Sanothimi Campus, } \\
\text { Tribhuvan University }\end{array}$} & \multirow{2}{*}{$\begin{array}{l}\text { MSc Edu (Biology) } \\
(\mathrm{S}: 10, \mathrm{~T}: 4)\end{array}$} \\
\hline & Plant taxonomy & & \\
\hline $\begin{array}{l}\text { Seminar } \\
16.02 .2013 \\
\text { NHM }\end{array}$ & $\begin{array}{l}\text { Ethnobiology } \\
\text { research } \\
\text { methods }\end{array}$ & $\begin{array}{l}\text { Golden Gate International } \\
\text { College, Tribhuvan } \\
\text { University (affiliation) }\end{array}$ & MSc Env Sci (S:24, T:1) \\
\hline \multirow[b]{2}{*}{$\begin{array}{l}\text { Seminar } \\
6.05 .2014\end{array}$} & $\begin{array}{l}\text { Bryophyte } \\
\text { collection }\end{array}$ & \multirow[b]{2}{*}{$\begin{array}{l}\text { Patan Multiple Campus, } \\
\text { Tribhuvan University }\end{array}$} & \multirow[b]{2}{*}{ BSc Env Sci (S:24, T:3) } \\
\hline & $\begin{array}{l}\text { Plant collection, } \\
\text { identification, } \\
\text { nomenclature } \\
\text { and } \\
\text { cclassification }\end{array}$ & & \\
\hline $\begin{array}{l}\text { Seminar } \\
\text { 21. } 05.2013 \\
\text { NHM }\end{array}$ & $\begin{array}{l}\text { NHM and } \\
\text { agroecotourism }\end{array}$ & $\begin{array}{l}\text { National College, } \\
\text { Kathmandu University } \\
\text { (affiliation) }\end{array}$ & B DevS (S:50,T:1) \\
\hline $\begin{array}{l}\text { International } \\
\text { Biodiversity Day } \\
22.05 .2013 \\
\text { ICIMOD, } \\
\text { Godawari }\end{array}$ & Plant taxonomy & $\begin{array}{l}\text { Padma High School, Kitini } \\
\text { High School and Padma } \\
\text { Kanya High School }\end{array}$ & $\begin{array}{l}\text { School children of } 7-10 \\
\text { Grades } \\
(\mathrm{S}: 30, \mathrm{~T}: 3)\end{array}$ \\
\hline $\begin{array}{l}\text { Workshop } \\
25.05 .2013 \\
\text { Taudaha }\end{array}$ & $\begin{array}{l}\text { Plant collection } \\
\text { techniques }\end{array}$ & $\begin{array}{l}\text { Community Children of Art } \\
\text { School, Chakupat }\end{array}$ & $\begin{array}{l}\text { Grade 1-9 } \\
\text { (S: 20, T: 2; P:5) }\end{array}$ \\
\hline $\begin{array}{l}\text { Class } \\
4.07 .2013 \\
\text { Rabibhawan }\end{array}$ & $\begin{array}{l}\text { Agrotourism in } \\
\text { Nepal }\end{array}$ & $\begin{array}{l}\text { Nepal Academy of Tourism } \\
\text { and Hotel Management } \\
\text { (NATHM) }\end{array}$ & $\begin{array}{l}\text { Master Students } \\
(\mathrm{S}: 24, \mathrm{~T}=2)\end{array}$ \\
\hline $\begin{array}{l}\text { Seminar } \\
11.08 .2013 \\
\text { NHM }\end{array}$ & $\begin{array}{l}\text { Plant collection } \\
\text { and herbarium } \\
\text { preparation }\end{array}$ & $\begin{array}{l}\text { Tri-Chandra Multiple } \\
\text { Campus, Tribhuvan } \\
\text { University }\end{array}$ & $\begin{array}{l}\text { B. Sc. students } \\
(\mathrm{S}: 22, \mathrm{~T}=1)\end{array}$ \\
\hline
\end{tabular}




\begin{tabular}{|c|c|c|c|}
\hline $\begin{array}{l}\text { Seminar } \\
7.09 .2013 \\
\text { Kirtipur }\end{array}$ & $\begin{array}{l}\text { Introducing } \\
\text { Natural History } \\
\text { Museum }\end{array}$ & $\begin{array}{l}\text { Mangal Higher Secondary } \\
\text { School }\end{array}$ & $\begin{array}{l}8^{\text {th }} \text { grade students } \\
(\mathrm{S}: 51, \mathrm{~T}=3)\end{array}$ \\
\hline $\begin{array}{l}\text { Class and field } \\
13.09 .2013 \\
\text { Ilam }\end{array}$ & $\begin{array}{l}\text { Weed diversity } \\
\text { (Theory); weed } \\
\text { collection and } \\
\text { identification } \\
\text { (practical) }\end{array}$ & $\begin{array}{l}\text { Mahendra Ratna Multiple } \\
\text { Campus, Ilam } \\
\text { Namsaling Community } \\
\text { Development Center, Ilam }\end{array}$ & $\begin{array}{l}\text { BSc Horticulture } \\
\text { \& Floriculture } \\
\text { Management } \\
(\mathrm{S}: 29, \mathrm{~T}: 2)\end{array}$ \\
\hline $\begin{array}{l}\text { Training } \\
21.09 .2013 \\
\text { Kirtipur }\end{array}$ & $\begin{array}{l}\text { Proposal } \\
\text { development }\end{array}$ & $\begin{array}{l}\text { NHM, Mangal Higher } \\
\text { Secondary School,Kirtipur }\end{array}$ & $\begin{array}{l}8^{\text {th }} \text { grade students } \\
(\mathrm{S}: 21, \mathrm{~T}=1)\end{array}$ \\
\hline $\begin{array}{l}\text { Training } \\
\text { workshop } \\
1-3.10 .2013 \\
\text { NHM }\end{array}$ & $\begin{array}{l}\text { Training on } \\
\text { environmental } \\
\text { education }\end{array}$ & NHM, IAAS, Schools & $\begin{array}{l}\text { Staff, students, school } \\
\text { teachers } \\
(\mathrm{St}: 5, \mathrm{~S}=5, \mathrm{~T}=10)\end{array}$ \\
\hline $\begin{array}{l}\text { Training } \\
15-17.11 .2013 \\
\text { ECCA office }\end{array}$ & $\begin{array}{l}\text { Training for } \\
\text { understanding } \\
\text { climate change }\end{array}$ & $\begin{array}{l}\text { Rotaract, ECCA } \\
\text { Counselors, NHM }\end{array}$ & $\begin{array}{l}\text { Staff, students, and } \\
\text { school teacher } 1 \\
(\mathrm{St}=2, \mathrm{~S}: \quad, \mathrm{T}=1)\end{array}$ \\
\hline $\begin{array}{l}\text { Seminar } \\
29.12 .2013 \\
\text { NHM }\end{array}$ & $\begin{array}{l}\text { Natural History } \\
\text { Museum }\end{array}$ & $\begin{array}{l}\text { Tri-Chandra Multiple } \\
\text { Campus, Tribhuvan } \\
\text { University }\end{array}$ & $\begin{array}{l}\text { B.Sc. Botany students } \\
\text { and teacher } \\
(\mathrm{S}: 31, \mathrm{~T}=1)\end{array}$ \\
\hline $\begin{array}{l}\text { Seminar } \\
2.02 .2014 \\
\text { NHM }\end{array}$ & $\begin{array}{l}\text { Wetland research } \\
\text { findings }\end{array}$ & $\begin{array}{l}\text { NHM, IAAS, CDES, Golden } \\
\text { Gate International College }\end{array}$ & $\begin{array}{l}\text { NHM staff, student } \\
\text { researchers, Dean, } \\
\text { Research division chief } \\
\text { of UGC }\end{array}$ \\
\hline $\begin{array}{l}\text { Seminar } \\
10.03 .2014 \\
\text { NHM }\end{array}$ & $\begin{array}{l}\text { Seminar series: } \\
\text { botanical } \\
\text { research } \\
\text { methods }\end{array}$ & $\begin{array}{l}\text { Students of Patan Multiple } \\
\text { Campus, Ascol \& Tri- } \\
\text { Chandra Multiple Campus }\end{array}$ & $\begin{array}{l}\text { Students and teachers } \\
(\mathrm{S}: 18, \mathrm{~T}=3)\end{array}$ \\
\hline $\begin{array}{l}\text { Seminar } \\
3.12 .2014 \\
\text { NHM }\end{array}$ & $\begin{array}{l}\text { Plant genetic } \\
\text { resources } \\
\text { education }\end{array}$ & NHM, Schools, Colleges & $\begin{array}{l}\text { NHM staff, school } \\
\text { teachers, College } \\
\text { students } \\
\text { ( } \mathrm{St}=12, \mathrm{loST}=2, \mathrm{~T}=9 \text {, } \\
\text { CENEED }=1, \mathrm{IAAS}=1 \text { ) }\end{array}$ \\
\hline $\begin{array}{l}\text { Seminar } \\
\text { 11.12. } 2014 \\
\text { NHM }\end{array}$ & $\begin{array}{l}\text { Seminar on } \\
\text { research findings }\end{array}$ & Different campuses of IOST & $\begin{array}{l}\text { Researchers, } \\
\text { NHM participants } \\
(\mathrm{St}=10, \mathrm{~T}=14, \mathrm{~S}=1)\end{array}$ \\
\hline
\end{tabular}




\begin{tabular}{|c|c|c|c|}
\hline $\begin{array}{l}\text { Seminar } \\
30.12 .2014 \\
\text { NHM }\end{array}$ & $\begin{array}{l}\text { Learning from } \\
\text { volunteering in } \\
\text { NHM by Salina } \\
\text { Maharjan }\end{array}$ & NHM, Sanothimi Campus & $\begin{array}{l}\text { NHM staff, teachers and } \\
\text { students of Sanothimi } \\
\text { Campus }\end{array}$ \\
\hline $\begin{array}{l}\text { Training } \\
29.01 .2015 \\
\text { Kirtipur }\end{array}$ & $\begin{array}{l}\text { Herbarium } \\
\text { preparation }\end{array}$ & $\begin{array}{l}\text { Mangal Higher Secondary } \\
\text { School }\end{array}$ & $\begin{array}{l}\text { Students and teachers } \\
(\mathrm{S}: 52, \mathrm{~T}=5)\end{array}$ \\
\hline
\end{tabular}

$\mathrm{ISt}=\mathrm{Staff}, \mathrm{S}=$ students, $\mathrm{T}$ : teachers, $\mathrm{P}:$ parents

Natural History Museum, the only of its kind in Nepal has good plant collections useful to different institutions for teaching and research. So, development and implementation plans, programs and projects in different districts of Nepal are needed to enrich specimens of both lower and higher plants. Only two botanists of NHM worked or have been working for more than 30 years. One had resigned 4 years before. Most of the employees worked for shorter period only. Collaborative research projects conducted by the botanists are few in numbers. Therefore, exploration of the potential of tangible national and international interactions for encouragement of networking, uni or multidisciplinary research work and online databases by means of new botanists are needed. This museum also has expertise in botanical research and training. So, more educational and training programs useful to academic and research organizations of Nepal and abroad can be developed to expand its horizon. Further, well experienced botanists of NHM can also play a pivotal role in designing credit courses and training programs for providing quality education and generating income for academic excellence. Lastly, Tribhuvan University, especially Institute of Science and Technology, needs to develop policy to utilize human resources and facilities currently present in Natural History Museum effectively in strengthening education and research capacity of institutions, faculty members, researchers and students of different departments, campuses and affiliated colleges of IOST.

\section{ACKNOWLEDGEMENTS}

Author is thankful to Prof. Dr. Bhaiya Khanal and Prof. Dr. Nirmala Pradhan of Natural History Museum for their helpful suggestions to improve the manuscript and NHM staffs (Krishna Bahadur Shrestha, Keshav Lal Shrestha, and Devendra Maharjan) for their help in collecting information for this article.

\section{REFERENCES}

Dangol, D R (2013) Hernaiparne Prakritik Bigyan Sangrahalaya. Gorakhapatra, Shanibar, Chaitra 3, 2069:6 (in Nepali).

Shrestha, K; Dahal B R (2010) A checklist of plants of historical collection of Bis Ram, Sharma, K N; Gupta, B L Journal of Natural History Museum 25: 155-200.

TU Today (2015) Natural History Museum. Tribhuvan University, Kirtipur, Kathmandu, Nepal; pp 31-32. 
Appendix 1. Publications authored by botanists of Natural History Museum, Tribhuvan University, Nepal.

\section{Abstracts (5)}

BhandARY, H R; Shrestha, P (1982) Ethnobotanical explorations on the poisonous plants of Kathmandu valley. Abstracts: Science and Technology Congress, NCST, Kathmandu, Nepal.

Pradhan, N; Joshi, S D (2002) Tropical bryoflora of Nepal. In SRIVAstaVa, R; Nath,V; WaHal, D; Asthana, A K; SAXenA, N K (eds) Abstracts: of 72, World Conference of Bryology. January 23-30, 2002, Lucknow, India: WCB 2002. National Botanical Research Institute, Lucknow, India.

Shrestha, I; Shrestha, K (2007) Medicinal and aromatic plants of Langtang National Park, Nepal. National Seminar on Sustainable Use of Biological Resources with the Special Theme: Medicinal and Aromatic Plants (April 22-23, 2007, Baishak 9-10, 2064, Pokhara, Nepal) Abstracts: 40-41. Ecological Society, Kathamndu, Institute of Forestry, Pokhara and PN Campus, Pokhara.

Shrestha-Vaidya, G; Shrestha, K; Wallander, H (2007) Evaluation of antimicrobial activity of Kande jhar (Lantana camara Linn.) against pathogenic fungi and bacteria. National Seminar on Sustainable Use of Biological Resources with the Special Theme: Medicinal and Aromatic Plants (April 22-23, 2007, Baisak 9-10, 2064, Pokhara, Nepal) Abstracts: 47-48. Ecological Society, Kathamndu, Institute of Forestry, Pokhara and PN Campus, Pokhara.

Siwakotı, M (2007) Wetlands conservation and sustainable livelihoods in Nepal. National Seminar on Sustainable Use of Biological Resources with the special theme: Medicinal and Aromatic Plants (April 22-23, 2007, Baisak 9-10, 2064, Pokhara, Nepal) Abstracts: 7-8. Ecological Society, Kathamndu, Institute of Forestry, Pokhara and PN Campus, Pokhara.

\section{Books and booklets (12)}

PRADHAN, N (2000) Materials for a checklist of bryophytes of Nepal-a catalogue of bryophyte specimens collected from Nepal. The Natural History Museum, London, UK; 79 pp.

ShRESTHA, K (1979) Nepali names for plants. Natural History Museum, Institute of Science, Tribhuvan University, Swayambhu, Kathmandu, Nepal; 38 pp.

SHRESTHA, K (1984) A field guide to Nepali names for plants. Natural History Museum, Institute of Science and Technology, Tribhuvan University, Kathmandu, Nepal; 156 pp (2nd edition).

Shrestha, K; Corvinus, G (2000) The Nepal Siwaliks: their environmental and palaeontological importance. Natural History Museum, Tribhuvan University, Swayambhu, Kathmandu, Nepal; 50 pp.

Shrestha, K; Khanal, B; Shah, K B; Shrestha, P K; Pradhan, N; Tiwari, S; Pandit, S (2059) Nepalka sanrachhyit danyajantu tatha danaspati CITES sandarvaka yek chinari. IUCN, Kathmandu, Nepal (in Nepali).

Shrestha, K; Khanal, B; Shrestha, P K; Shah, K B; Pradhan, N; Timari, S; Pandit, S (2002) Nepalka samrakchhit biruwaharu ra janawarharu (Protected plants and animals of Nepal). Natural History Museum, Tribhuvan University, Swayambhu, Kathmandu, Nepal (in Nepali). 
Shrestha, K; Budhathoki, P; Kazi, H S N; Verhengt, W J M (1995) Biodiversity assessment of forest ecosystems of the central mid-Hills of Nepal. Technical Publication No. 8, Department of National Parks and Wildlife Conservation, Ministry of Forests and Soil Conservation, His Majesty's Government of Nepal, Kathmandu, Nepal; 146 pp.

SINGH, S C (1975) Collecting and preserving plants (mimeograph). Tribhuvan University, Institute of Science, Natural History Museum, Anandakuti, Swayambhu, Kathmandu, Nepal; 6 pp.

SINGH, S C (1976) Collecting and preserving plants (mimeograph). Tribhuvan University, Institute of Science, Natural History Museum, Anandakuti, Swayambhu, Kathmandu, Nepal; 5 pp.

SINGH, S C (1978) Plan and policy of Natural History Museum (mimeograph). Tribhuvan University, Institute of Science, Natural History Museum, Swayambhu, Kathmandu, Nepal; $21 \mathrm{pp}$.

SINGH, S C (1979) Natural History Museum: objectives, programmes and activities (1975 July 1979 June) (mimeograph). Tribhuvan University, Institute of Science, Natural History Museum, Anandakuti, Swayambhu, Kathmandu, Nepal; 42 pp.

Timarı, S; Adhikari, B; Siwakotı, M; Subedi, K (2005) An Inventory and assessment of invasive alien plant species of Nepal. IUCN, Kathmandu, Nepal.

\section{Edited book chapters (10)}

Bhandary, H R (1999) Mushrooms. In Majupuria, TC; Majupuria, R K (eds) Nepal nature's paradise. DeVI, M, Gwailior, India; pp 235-246.

Bhandary, H R; Shrestha. P (1999) Poisonous plants of Kathmandu valley. In Majupuria, TC; MAJPURIA, R K (eds) Nepal nature's paradise. DevI, M, Gwailior, India; pp 151-158.

Dangol, D R (2015) Plant communities and local uses: observations from Chitwan National Park, Nepal. In Dhakal, M; Shrestha, R (eds) Biodiversity conservation efforts in Nepal, special issue, 2072 (2015). Department of National Parks and Wildlife Conservation (DNPWC), Kathmandu, Nepal; pp 85-93.

Dangol, D R (2015) Status of weed science in Nepal. In Rao, V S; Yaduraja, N T; Chandrasena, N R; HASAN, G; Sharma, AR (eds) Weed science in Asian Pacific Region. Asian Pacific Weed Science Society and Indian Weed Science Society, India; pp 305-322.

Pradhan, N (2003) Results from a questionnaire on bryophytes from Nepal. Species Survival Commission (SSC), Worldwide Status of Bryophyte Conservation, Sweden; pp 50-51.

Pradhan, N; Joshi, S D (2007) Tropical bryoflora of Nepal. In Nath, V; Asthana, A K (eds) Current trends in bryology. Bishen Singh \& Mahendra Pal Singh, Dehradun, India; pp 17-36.

Pradhan, N (2013) Biodiversity: Bryophyta. In Jha, P K; Neupane, F P; Shrestha, M L; Khanal, I $P$ (eds) Biological diversity and conservation. Nepalpedia series 2. Nepal Academy of Science and Technology, Khumaltar, Lalitpur, Nepal; pp 113-117.

Shrestha (Pradhan), I; Shrestha. K (2007) Ethnobotanical diversity (Gosainthan). In Bhandari, B B; JOO, G J (eds) Gosainthan: a secret wetland in Nepal. Nepal Wetland Society, Kathmandu, 
Nepal; pp 60-62.

Siwakotı, M (2007) Flora of the Gokyo. In BhAndarı, B B; JOO, G J (eds) Himalayan wetlands: risks, challenges and opportunities. Changwon ramsar wetlands center, Korea; pp 43-51.

SIWAKOTI, M (2007) Floral diversity of the Gosainkund wetland system. In BHANDARI, B B; JOO, G J (eds) Gosainthan: a sacred wetland in Nepal. Nepal Wetland Society, Kathmandu, Nepal; pp 55-59 and 92-96.

\section{Journal and bulletin articles (105)}

Abe, H; Shrestha, K; Shrestha, P K (2001) Water mites (Acari: Hydrachnellae) in Kathmandu valley, Nepal. Journal of Natural History Museum 20: 173-177.

AdHIKARI, M K; Shrestha, K (2011) New records of some higher fungi (mushrooms) from Nepal. Bull. Dept. PI. Res. 33: 12-16.

AdHIKARI, R; SHRESTHA, K (2008) Intraspecific variation of Bambusa nutans subsp. nutans from six different sites of central Nepal. Scientific World 6(6): 81-84.

Adhikari, R; Shrestha, K; SAKya, S R (2006) Morphological variation of Bambusa nutans subspecies nutans from six different sites of central Nepal. Scientific World 4(4): 71-73.

AdHIKARI, R; SHREStha, K; MANANDhAR, M D (2006) Variation in the total phenolic compounds in the Bambusa nutans subspecies nutans found in central Nepal. Bamboo Journal 23: 37-41.

Ale, R; Raskoti, B B; Shrestha, K (2009) Ethnobotanical knowledge of Magar community in Siluwa VDC, Palpa district, Nepal. Journal of Natural History Museum 24: 58-71.

Bhandary, H R (1980) Notes on some macrofungi from Nepal. Journal of Natural History Museum 4(1-4): 23-32.

BHANDARY, HR (1991) Some edible and medicinal fungi from Dumre to Manang, Mustang and Pokhara. Journal of Natural History Museum 12(1-4): 47-59.

Bhandary, H R; Shrestha, P (1982) Ethnobotanical approach on the poisonous plants of Annapurna and Langtang Himal area. Journal of Natural History Museum 6(1-4): 125-135.

BhANDARY, H R; SHREstha, P (1986) Ethnobotanical investigations on the poisonous plants of Manang-Mustang and adjoining areas. Journal of Natural History Museum 10(1-4): 133-144.

Bhandary, H R; Shrestha, P; Bhattaral, T B (1982) Nutrient content in the leaves of ten plants used as green manure in Kathmandu valley. Journal of Institute of Science and Technology 5: 57-62.

Bhandary, H R; Shrestha, P; Bhattaral, T B (1982) Nutrient content in the leaves of the plants used as green manure in Kathmandu valley. Journal of Institute of Science and Technology 5: 57-82.

Bhattarai, S M; Dangol, D R; Srivastav, S B; Shrestha, P K (2015) Factors influencing local innovation in ecological agriculture in the central development region of Nepal. Nepalese Journal of Agricultural Sciences 13: 69-84. 
BhuRtYal, S; Dangol, D R; Joshi, A R (2015) Assessing local practices of organic vegetable production in Bhadaure Tamagi, Kaski, Nepal. The Journal of Agriculture and Environment 16: 132-141.

Cotter, H; van, T; Bhandary, H R (1985) Cavimalum indicum (Clavicipitaceae) on Arundinaria in Nepal. Journal of Natural History Museum 6(1-4): 115-120.

DANGoL, D R (2013) Weeds of wheat in Nepal: a literature review. Journal of Natural History Museum 27: 132-178.

Dangol, D R; Khanal, S (2013) Status of "Wetland Ecology" education and research at the Department of Environmental Science, Institute of Agriculture and Animal Science of the Tribhuvan University, Nepal. Nepalese Journal of Biosciences 3: 78-86.

Dangol, D R; Gautam , B; Oli, B B (2014) Wetland plants and their local uses: observations from Rampur ghol, Chitwan, Nepal. Journal of Natural History Museum 28: 142-159.

Dangol, D R; Shrestha, Y M; Joshi, N (2015) Weed flora of llam Tea Estate, east Nepal. Himalayan Biodiversity 3: 1-11.

Gautam, B; Maskey, R; Sapkota, R; Dangol, D R (2014) Aquatic macro-invertebrates as bioindicators: an approach for wetland water quality assessment of Rampur ghol, Chitwan. Journal of Institute of Science and Technology 19(2): 58-64

Karki, J B; Shrestha, K; Khanal, B (2003) Faunal diversity and related conservation issues at Badimalika region (Achham, Bajura and Kalikot districts), Nepal. The Wildlife 14-22 pp.

KHANAL, B; BhANDARY, H R (1982) Food plants of some butterfly larvae. Journal of Natural History Museum 6(1-4): 57-69.

Khanal, B; Shrestha, K (2000) Habitat preferences by Royles pika (Ochotona roylei) in Gosainkund, Rasuwa district of central Nepal. Journal of Natural History Museum 19: 27-33.

KHANAL, S; Dangol, D R (2014) Identification and interaction of stakeholders for socio-ecological system in Bishajari lake and associated wetland area, Chitwan, Nepal. Himalayan Biodiversity 2: $15-24$.

Khanal, S; Gurung, S B; Pant, K K; Chaudhary, P; Dangol, D R (2014) Ecosystem services and stakeholder analysis in Bishajari lake and associated wetland areas, Chitwan, Nepal. International Journal of Applied Sciences and Biotechnology 2(4): 563-569.

Konda, T; Nakanishi, M; Sako, Y; Ishida, Y; Shrestha, K; Bhandary, H R; Shrestha, R L (1988) Bacterial numbers and floras in the water and sediment of subtropical lakes Phewa, Begnas and Rupa in Nepal. Bulletin of Japanese Society of Microbial Ecology 3(1): 21-28.

Kunwar, R M; Shrestha, K; Dhungana, S K; Shrestha, P R; Shrestha, K K (2010) Floral biodiversity of Nepal: an update. Journal of Natural History Museum 25: 295-311.

Kunwar, R M; . Shrestha, K P; Bussmann, E (2010) Traditional herbal medicine in the far-west Nepal: a pharmacological appraisal. Journal of Ethnobiology and Ethnomedicin 6: 35.

Kunwar, R M; Shakya, S; Shrestha, K (2005) Diversity and relationship study of flora and 
avifauna in Babai river valley, Bardia Nepal. Journal of Natural History Museum 22: 64-70.

Nakanishi, M; Watanabe, M M; Terashima, A; Sako,Y; Konda, T; Shrestha, K; Bhandary, H R; ISHIDA, Y (1988) Studies on some limnological variables in subtropical lakes of the Pokhara valley, Nepal. Japanese Journal of Limnology 49(2): 71-86.

Nisha; Singh, S C; Joshi, A R (1977) Myxomycetes of Nepal -III. Journal of Natural History Museum 1(2-4): 215-219.

Oli, B B; Jha, D K; Aryal, P C; Shrestha, M K; Dangol, D R; Gautam, B (2013) Seasonal variation in water quality and fish diversity of Rampur ghol, a wetland in Chitwan, central Nepal. Nepalese Journal of Biosciences 3: 9-17.

Pradhan, N (2000) Bryophytes of Phulchoki, central Nepal. Journal of Natural History of Museum 19: 57-81.

Pradhan, N. (2001) Contribution to the bryoflora of Swayambhu, central Nepal. Journal of Natural History of Museum, 20: 25-38.

PradHAN, N (2002) Species richness of bryoflora of Royal Bardia National Park, midwestern Nepal. Journal of Natural History of Museum 21: 45-60.

PRADHAN, N (2013) Diversity and status of bryophytes in Panch Pokhari Region of the northern Sindhupalchok district of Central Nepal. Journal of Natural History Museum 27: 45-58.

Pradhan, N (2014) Altitudinal distribution of bryoflora at Chandragiri mountain forest of Kathmandu district, central Nepal. Journal of Natural History Museum 28: 81-92.

PRADHAN, N (2014) Three new records of Jungermannia species (Hepaticae, Jungermanniales) from Nepal. International Journal of Environment 3(1): 28541-28548.

PRADHAN, N; JoshI, S D (1986) Contribution to the flora of liverworts of Kathmandu valley. Journal of Natural History of Museum 10(1-4): 73-82.

Pradhan, N; Joshi, S D (2006) A checklist of Fissidens species (Musci: Fissidentaceae) of Nepal. Our Nature 4(1): 61-68.

Pradhan, N; Joshi, S D (2007) Fissidens robinsonii Broth. (Fissidentaceae, Musci), a new record for Nepal. Geobios 34(2-3): 105-108.

Pradhan, N; Joshi, S D (2007) Species diversity of hornworts (Anthocerotae, Bryophytes) in lowland Nepal with an account of Folioceros assamicus D.C. Bhardwaj, a new report to the country. Our Nature 5: 31-36.

Pradhan, N; Joshi, S D (2008) A diversity account of Bryaceae (Bryophyta: Musci) of Nepal. Journal of Natural History of Museum 23: 19-26.

PradHAN, N; Joshi, S D (2008) The species composition and distribution of bryoflora in the inner valley of Dang district, west Nepal. Nepal Journal of Plant Sciences 2: 27-34.

PRADHAN, N; JoshI, S D (2009) Liverworts and hornworts of Nepal: a synopsis. Botanica Orientalis- The Journal of Plant Science 6: 69-75.

Pradhan, N; Joshi, S D (2009) Notes on Heteroscyphus (Tayl.) Schiffn. (Geocalycaceae: 
Jungermanniales) of the lowland Tarai, Nepal. Journal of Natural History of Museum 24: 113-120.

Pradhan, N; Long, D G; Joshi, S D (2007) Monosolenium tenerum Griff. (Marchantiopsida, Monosoleniaceae) in Nepal. Cryptogamie Bryologie 28(3): 243-248.

Raskoti, B B; Ale, R; Shrestha, K (2012) Peristylus intrudens (Ames) Ormerod (Orchidaceae): A new record for the Flora of Nepal. Journal of Japanese Botany 87(2): 137-139.

SAKo, Y; Shrestha, K; UCHIDA, A; IshidA, Y; Salto, S (1991) Isozyme analysis of mating populations of Gonium pectorale (Chlorophyta). Journal of Phycology 27(2): 309-315.

Sako, Y; Nakanishi, M; Konda, T; Ishida, Y; Kadota, H; Shrestha, K; Bhandary, H R; Shrestha, $\mathrm{R} L$ (1986) Life cycle of Peridinium sp. B3 (Dinophyceae) isolated from lake Begnas, Nepal. Bulletin of the Japanese Society of Microbial Ecology 1(1): 19-27.

SHRESTHA, A M (1985) Distributional analysis of plant species from Suryabinayak forest area (Kathmandu). Journal of Natural History Museum 9(1-4): 75-92.

SHRESTHA, B K; DANGol, D R (2014) Impact of Mikania micrantha H.B.K. invasion on diversity and abundance of plant species of Chitwan National Park, Nepal. Journal of Institute of Science and Technology 19(2): 24-29.

Shrestha, I; Shrestha, K (2004) Some wild edible plants of Langtang National Park, Rasuwa district, central Nepal. Bulletin of Pure and Applied Sciences 23B(1): 35-45.

Shrestha, I; SHRESTHA, K (2005) Ethnobotanical notes on some ferns and fern allies of Langtang National Park, central Nepal. Nepal Journal of Plant Sciences 1: 124-128.

SHRESTHA, I; JoshI, N; SHRESTHA; K (2002) Report on some plants from Langtang National Park, Nepal. Journal of Natural History Museum 21: 71-92.

SHRESTHA, K (1983) Some high altitude lichens of Nepal. Journal of Natural History Museum 7(1-4): 15-23.

SHRESTHA, K (1983) Wild leafy and fruity vegetables consumed by the local inhabitants of Dharan. Journal of Natural History Museum 7(1-4): 35-42.

SHRESTHA, K (1984) Food habits of some wild mammals in Royal Chitwan National Park. Journal of Natural History Museum 8(1-4): 67-78.

Shrestha, K (1984) Grasses and sedges-Their ecology, distribution and economic uses in Manang-Mustang region. Journal of Natural History Museum 8(1-4): 41-53.

Shrestha, K (1985) Cordyceps nutans Pat. from Lato Manang. Journal of Natural History Museum 9(1-4): 111-114.

SHRESTHA, K (1987) Report on edible wild plants from Pokhara and its northern region. Journal of Natural History Museum 11(1-4): 85-98.

SHRESTHA, K (1991) Seasonal changes in the food habits of Rhesus monkeys (Macaca mulata) in Swayambhu area, Kathmandu, Nepal. Journal of Natural History Museum 14(1-4): 33-46.

SHRESTHA, K (2000) A note on biochemical analysis of some vegetables found in Dharan, 
Nepal. Journal of Natural History Museum 19: 155-160.

ShresthA, K (2003) Natural History Museum: Present and future plans. Botanica Orientalis Annual Issue: 153-158.

SHRESTHA, K (2005) Orchids and their conservation at Natural History Museum, Kathmandu, Nepal. Journal of Natural History Museum 22: 57-63.

ShRESTHA, K (2006) Plant diversity, ethnobotany and conservation issues at Swoyambhu World Heritage, Kathmandu, Nepal. Nepal Journal of Science and Technology 7: 123-133.

SHRESTHA, K; DAHAL, B R (2010)A checklist of plants of historical collection of Bis Ram, SHARMA, K N; GuptA, B L Journal of Natural History Museum 25: 155-200.

Shrestha, K; Ghimire, G P S (1981) A comparative study on productivity of Trifolium repens L. and Imperata cylindrica (L.) Beauv. in Kirtipur. Journal of Institute of Science and Technology 4: 45-54.

ShRESthA, K; Kase, N (1997) Taxonomy and ethnobotany of Luffa graveolens Roxb. in Rana Tharu village of Kanchanpur district, Nepal. Journal of Natural History Museum 16: 62-69.

SHRESTHA, K; TIWARI; N N (2002) New report of Adenanthera pavonina Linn. and its ethnobotany in Nepal. Journal of Natural History Museum 21: 61-66.

Shrestha, K; KHANAL; B; KARKI, J B (1999) Foraging and haying plants of Royles pika (Ochotona roylei: Lagomorpha) in farwest Nepal. Journal of Natural History Museum 19: 3-13.

Shrestha, K; Wilson, E; GAy, H (2008) Ecological and environmental study of Eupatorium adenophorum Sprengel (Banmara) with reference to gall formation in Gorkha-Langtang route, Nepal. Journal of Natural History Museum 23: 108-124.

Shrestha, K; PAndey, N; Kase, N (1999) Tropical flora in Royal Suklaphanta Wildlife Reserve. Journal of Natural History Museum 18: 31-44.

Shrestha, K; SAKo, Y; IshidA, Y (1993) A note on isozyme analysis of $F_{1}$ progeny of Gonium pectorale. Bulletin of Japanese Society of Microbial Ecology 8(1): 43-46.

SHRESTHA, K; SAKo, Y; ISHIDA, Y (1993) Intraspecific crossing, zygote, germination and $F_{1}$ progeny of Gonium pectoral. Bulletin of Japanese Society of Microbial Ecology 8(1): 35-42.

ShRESTHA, K; SAKo,Y; ISHIDA, Y; UCHIDA, A (2001) Antagonistic effect and partial purification of lethal substance in Tibetan stains of Gonium pectorale. Journal of Natural History Museum 20: 13-24.

Shrestha, P K; Shrestha, K (2006) Ento-floral study in tropical Bardia and Karnali districts, mid-western region of Nepal. Scientific World 4(4): 60-70.

Shrestha, P K; Pradhan. N (1997) Beetles of Phulchoki hill and their food plants. Journal of Natural History of Museum 16(1-4): 1-16.

Shrestha, P K; Khanal, B; Shrestha, K (2002) Insect diversity of lowerbelt of Karnali region, mid west Nepal. Journal of Natural History Museum 21: 67-78.

Shrestha, P K; Pradhan, N; Shrestha, K (2001) Some insects and their food plants in the Royal 
Bardia National Park, mid-western Nepal. Journal of Natural History of Museum 20: 39-56.

SHRESTHA, S D (1977) Some liverworts of Nepal. Journal of Natural History Museum 1(2-4): 183-202.

Shrestha-Vaidhya, G; Shrestha, K; Wallander, H (2008) Effect of plant residues on AM fungi. Scientific World 6(6): 85-88.

Shrestha-Vaidya, G; SHREstha, K (2005) Performance of Pinus roxburghii inoculated with pure culture of four indigenous ectomycorohizal fungi. Nepal Journal of Science and Technology 6: 41-45.

Shrestha-Vaidya, G; Shrestha, K; Wallander, H (2005) Antagonistic study of ectomycorrhizal fungi isolated from Baluwa forest (central Nepal) against with pathogenic fungi and bacteria. Scientific World 3(3): 49-52.

Shrestha-Vaidya, G; Shrestha, K; Wallander, H (2007) Function of organic matter (green manure) and the effect on soil properties. Banko Jankari 17(2): 62-69.

Shrestha-Vaidya, G; Shrestha, K; Khadge, B R; Johnson, N C; Wallander, H (2007) Study of biodiversity of arbuscular mycorrhizal fungi in addition with different organic matters in different seasons of Kavre district (central Nepal). Scientific World 5(5): 75-80.

Shrestha-Vaidya, G; Shrestha, K; Khadge, B R; Johnson, N C; Wallander, H (2008) Organic matter stimulates bacteria and arbuscular mycorrhizal fungi in Bauhinia purpurea and Leucaena diversifolia plantations on eroded slopes in Nepal. Restoration Ecology 16(1): 79-87.

Shrestha-Vaidya, G; Shrestha, K;Wallander, H; . Khadge, B R (2006) Study of rhizospheric soil microflora of Baluwa forest of Kavre district (central Nepal) Scientific World 4(4): 44-49.

Shrestha-Vaidya, G; Thapa, S; Shrestha, A; Shrestha, K (2006) Antibacterial activity of the wild mushrooms against human pathogens. Nepal Journal of Science and Technology 7: 55-58.

SINGH, S C (1979) The Natural History Museum of Nepal, Katmandu. Museum 31(4): 219-228.

SINGH, S C; JosHI, A R (1977) Bibliography on fungi of Nepal. Journal of Natural History Museum 1(2-4): 249-254.

SINGH, S C; UpADHYAY. B N (1978) Note on some fungi new to Nepal. Journal of Natural History Museum 2(1-4): 51-55.

SINGH, S C; AdHIKARI, M K (1977) Some fleshy fungi of Kathmandu (Nepal). Journal of Natural History Museum 1: 49-57.

SingH, S C; NisHA (1976) Myxomycetes of Nepal -II. Journal of Science (Trichandra College Science Association) 6(2-4): 65-72.

SINGH, S C; SHARMA, G P; NisHa (1977) Notes on Blastocladia. Journal of Natural History Museum 1: 59-62.

Singh, S C; Bhandary, H R; Shrestha, P K; Shrestha, K; Pradhan , N (1997) A note on natural history data base in progress. Journal of Natural History Museum 16: 70-72.

SINGH, S C; SHRESHA, K; BHANDARY, HR (1996) Germination behaviour of some rare and endemic 
plants of Nepal. Journal of Natural History Museum 15: 1-12.

Siwakotı, M (2005) Lippia alba (Mill.) Britton ex Britton \& Wilson, a new addition to the Flora of Nepal. Journal of Natural History Museum 22: 157-158.

Siwakotı, M (2006) An overview of floral diversity in wetlands of Terai region of Nepal. Our Nature 4: 83-90.

Siwakotı, M (2007) Mikania weed: A challenge for conservationists. Our Nature 5: 70-74.

SıWAкотı, M (2007) Wetland types and associate vegetation in Nepal: an overview. Wetland Science (China) 5(3): 193-200.

SIWAKOTI, M; TIWARI, S (2007) Emerging needs of wetlands protection for the conservation of wild rice biodiversity in Nepal: a case study from Lumbini area. Scientific World 5:95-99.

Siwakotı, M; ShIVAKotı, K P; Karki, B; Siwakotı, S (2005) Ethnobotanical uses of plants among Rajbanshi and Dhimal ethnic communities of eastern Nepal. Journal of Natural History Museum 22: 41-56.

UpADHYAYA, B N (1977) Albugo bliti on Alternanthera sessilis in Nepal. Journal of Natural History Museum 1(2-4): 247-248.

UpADHYAYA, B N (1979) Two new records of Oscillatoria for Nepal. Journal of Natural History Museum 3(1-4): 73-74.

\section{Proceedings papers (19)}

Gautam, I; Pradhan, N; Khanal, B (2013) Empowering biological science in 21st century. Proceedings of the Seminar on Empowering of Biological Science in 21st Century in Nepal (April 30, 2013). Nepal Academy of Science and Technology, Khumaltar, Lalitpur, Nepal; pp 25-32.

KARKI, J; SiWAKotI, M; SHRESTHA, N (2005) Biodiversity resources of the high altitude wetlands in Nepal: Field studies of the Gosainkund-Naukund and Gokyo wetlands. In High altitude wetlands of Nepal, Views and Reviews on Conservation-The Proceedings of the National Workshop on High Altitude Wetlands of Nepal (2005). Forum for Ecosystem Management, Kathmandu, Nepal; pp 67-86.

Khanal, B; Shrestha , P K; Shrestha, K (2003) Distribution of butterflies in Parsa district, Nepal. In Neupane, F P; Bajracharya, K M; Bhuju, D R (eds) Proceedings of International Seminars on Mountains-Kathmandu, March 6-8, 2002. Royal Nepal Academy of Science and Technology, Kathmandu, Nepal; pp 590-594.

Pradhan, N; Shrestha, K (2002) Bryophytes: neglected plant resources in Nepal. In: Watanabe, T; Takano, A; BIsta, M S; SAIJU, H K (eds) Proceedings of Nepal-Japan Joint Symposium on Conservation and Utilization of Himalayan Medicinal Resources, November 6-11, 2000, Kathmandu, Nepal; pp 237-241.

Pradhan, N; Shrestha, K (2003) Alpine bryoflora of Nepal. In Neupane, F P; Bajracharya, K M; Bhusu, D R (eds) Proceedings of International Seminar on Mountains-Kathmandu, March 6-8, 2002. Royal Nepal Academy of Science and Technology, Kathmandu, Nepal; pp 545-562. 
Pradhan, N; Simakoti, M; Khanal, B; Bhattral, S; Thapa, G; Thapa, V (2012) Assessment of biodiversity in Panch Pokhari of Sindhupalchok district of central Nepal. Nepalese Conference for Rufford Grantees (January13-14, 2012.); pp 127-129.

SHRESTHA, I; Shrestha, K (2000) Ethno-medico-botanical studies of Langtang National Park, Nepal. In Watanabe, T; Takano, A; Bista, M S; SalJu, H K (eds) Proceedings of Nepal-Japan Joint Symposium on Conservation and Utilization of Himalayan Medical Resources, November 6-11, 2000, Kathmandu, Nepal; pp 178-181.

Shrestha, I; Shrestha, K (2006) Medicinal plants in ethno veterinary practices in Langtang National Park, Nepal. In Füsun ERTU, Z (ed) Proceedings of the Fourth International Congress of Ethnobotany (ICEB 2005) 21-26 August 2005. Yeditepe University, Istanbul, Turkey; pp 277-280.

Shrestha, I; Inglis, A; Shrestha, K (2003) Documentary and mapping medicinal plants of Langtang National Park, central Nepal. In Neupane, F P; BajRacharya, K M; Bhuju, D R (eds) Proceedings of International Seminar on Mountains-Kathmandu, March 6-8, 2002. Royal Nepal Academy of Science and Technology, Kathmandu, Nepal; pp 361-371.

SHRESTHA, K (1991) Bamboo of Kathmandu. Proceedings of Kyoto Profecture University, Japan.

SHRESTHA, K (1998) Distribution and status of bamboos in Nepal: Conservation, diversity, ecogeography, germplasm resource utilization and taxonomy. Proceedings of Training Course and Workshop, 10-17 May; Kunming and Xinshunghaba, Yunan, China, www. biodiversityinternational.org/publications/web_version/572/ch29.htm-50k-; 215 pp.

SHRESTHA, K (2000) Major identification keys and ethnobotany of some of the bamboos of Sunsari district, east Nepal. Proceedings of Third National Conference on Science and Technology, March 8-11, 1999. Royal Nepal Academy of Science and Technology, Kathmandu, Nepal; pp 1535-1555.

SHRESTHA, K (2000) Observation on the germination of some seeds of medicinal plants of Nepal. In WATANABE, T; TAKANO, A; BISTA, M S; SAIJU, HK (eds) Proceedings of Nepal-Japan Joint Symposium on Conservation and Utilization of Himalayan Medical Resources, November 6-11, 2000, Kathmandu, Nepal; pp 201-207.

Shrestha, K (2009) Flowering and fruiting behavior of Melocanna baccifera in Nepal. Proceedings of the $8^{\text {th }}$ World Bamboo Congress, September 16-20, 2009. Thailand.

Shrestha, K; Khanal, B; KarkI, J B (2003) Floral diversity in Badmalika and Rama-Roshan region, mid-west Nepal. In: Neupane, F P; BajRacharya, K M; Bhuju, D R (eds) Proceedings of International Seminar on Mountains, Kathmandu, March 6-8, 2002. Royal Nepal Academy of Science and Technology, Kathmandu, Nepal; pp 578-589.

SHRESTHA, K; SHRESTHA, P K; KHANAL, B (2006) Ento-floral relation for food and their conservation issues in Parsa region of Central Nepal. In JHA, P K; CHAUDHARY, R P; KARMACHARYA, S $\mathrm{B} ; \mathrm{PRASAD}, \mathrm{V}$ (eds) Environment and plants-a glimpse of research in south Asia. Proceedings of Regional Seminar on Natural Resources and Management in Nepal. Birgunj Campus and Ecological Society, Kathmandu, Nepal; pp 155-168.

Shrestha, K; Shrestha, P K; Khanal, B (2006) Study of ento-floral relation for food and their 
conservation issue in Koshi region of east Nepal. In KARMACHARYA, S B; DHAKAL, M R; JHA, S N; Mandal, T N; Niroula, B; Chhetri, M K; Subba, B R; KoIrala, U; Limbu, K P (eds) Proceedings of the National Seminar on Natural Resource Management. Morang Campus, Tribhuvan University, Nepal; pp 116-128.

Shrestha, P K; Shrestha, K; Khanal, B (2003) Distribution of coleopteran fauna in Koshi zone, east Nepal and their conservation measures. In Neupane, F P; BajRacharya, K M; Bhuju, D R (eds) Proceedings of International Seminar on Mountains-Kathmandu, March 6-8, 2002. Royal Nepal Academy of Science and Technology, Kathmandu, Nepal; pp 499-507.

SIWAKOTI, M (2008) Medicinal and edible plants in wetlands of Nepal. In JHA, P K; KaRMACHARYA, S B; ChHetri, M K; ThapA, C B; Shrestha, B B (eds) Medicinal plants in Nepal: an anthology of contemporary research. Ecological Society, Kathmandu, Nepal; pp 130-138.

\section{Research/project reports (19)}

DANGOL, D R (2015) Establishment of plant genetic resources display and information center at Natural History Museum. An initiative of Institute of Science and Technology and Local Initiatives for Biodiversity, Research and Development with the support of Bioversity International, Rome (July 2014-Feb 2015).

Hagiwara, H; Bhandary, H R (1982) Myxomycetes from central Nepal I. Reports on the cryptogamic study in Nepal, March 1982 (Miscellaneous Publication of the National Science Museum, Tokyo, Japan); pp 119-124.

KARKI, J B; ShRESTHA, K; KHANAL, B (1998) Study on potential of biodiversity conservation of Badimalika region of Achham, Bajura, Bajhang and Kalikot districts of Nepal. A report submitted to Nepal Biodiversity Action Plan, Kathmandu.

Khanal, B; Shrestha, P K; Shrestha, K (2001) Study of insect fauna of tropical region of Nepal and their conservation measures. A final report submitted to Pro-Natura Fund, Japan/NCSJ, 134+Maps+photographs.

PRADHAN, N (2004) Bryoflora of Chitwan, central Nepal. Final report on study of the biodiversity of tropical region of Nepal (Chitwan district). A report submitted to Pro-Natura Fund, Japan; pp 48-61.

Pradhan, N; Shrestha, K; Shah, K B; Shrestha, P K; Khanal, B (2000) Study on the biodiversity of Swayambhu area. A report submitted to the Institute of Science and Technology, Tribhuvan University, Kathmandu, Nepal.

Shrestha, K; Shrestha, P K; Khanal, B; Shakya, S (2004) Study of the biodiversity of the tropical region of Nepal: a mid term report submitted to Pro-Natura Foundation, Japan.

Shrestha, K; Adhikari, B; Allen, M; Courage, A; Hall, H; Khanal, B; Koirala, S; Kunwar, R; Malicky, H; Pariya, B; Pradhan, N; Shah, K; Shakya, S; Sharma, S; Shaw, A; Shrestha, K; Shrestha, P K; SMith, C; Zimmerman, B (2003) Babai river valley fish and biodiversity survey: higher plants. In OLIVER, S (ed) Babai River Valley fish and biodiversity survey: Royal Bardia National Park, Nepal, March 2003 (ZSL Conservation Report), Zoological Society of London; 
pp 39-48.

Shrestha, K; Khanal; B; KarkI, J B (1998) Inventory of biodiversity in Badimalika and RamaRoshan region, west Nepal. A report submitted to National Biodiversity Action Plan, Kathmandu.

Shrestha, K; Khanal, B; Shrestha, P K (2001) Insect fauna and their conservation in tropical Nepal. Pro-Natura Fund, Japan; 10: 145-159.

Shrestha, K; Khanal, B; Karki, J B (2001) Floral diversity in Badimalika and Rama-Roshan region, mid-west Nepal. Natural History Museum, Tribhuvan University, Nepal/ Department of National Parks and Wildlife Conservation, His Majesty Government of Nepal.

Shrestha, K; Shrestha, P K; Khanal, B; Pradhan, N; Shakya, S (2005) Study on the species diversity in the Chitwan district of central Nepal. Annual report, Pro-Natura Fund 14: 175-192.

Shrestha, K; Shrestha, P K; Khanal, B; Pradhan, N; Shakya, S (2004) Study of the biodiversity of tropical region of Nepal (Chitwan district). A final report submitted to Pro-Natura Foundation, Japan submitted by CENEED/NHM, Tribhuvan University, Nepal.

SHRESTHA, K (2043) Study of the natural environment of Dang. A report submitted to RONAST, Nepal.

Shrestha, P K; Pradhan, N (2000) A general survey of invertebrate (except Lepidoptera) in Royal Bardia National Park, Nepal. A report submitted to World Wildlife Fund (WWF) Nepal Program, Kathmandu, Nepal.

Shrestha-Vaidya, G; Shrestha, K; Wallander, H (2008) Evaluation of the antimicrobial activity of Lantana camara Linn. against pathogenic fungi and bacteria. Nepal Academy of Science and Technology (NAST), Khumaltar, Lalitpur, Nepal and Microbial Ecology Lab, Lund University, Lund, Sweden.

SINGH, S C; BHANDARY, H R (1997) Establishment of computerized data base in the Natural History Museum. Draft report, Natural History Museum, Tribhuvan University, Swayambhu, Kathmandu, Nepal.

Singh, S C; SHRESTHA, K; BhANDARY, HR (1996) A study of some rare, endangered and endemic plants of central Nepal. Draft report, Natural History Museum, Tribhuvan University, Swayambhu, Kathmandu, Nepal.

Wilson, E; Walisiewicz, M; Harvey, S; Gay, H; Shrestha, K (1985) The Report of the Oxford University expedition to Nepal 1985 (Unpublished). 